\title{
SUSY-Induced Top Quark FCNC Processes at Linear Colliders
}

\author{
Junjie $\mathrm{Cao}^{a, b}$, Zhaohua Xiong ${ }^{a, c}$ and Jin Min Yang ${ }^{b}$ \\ ${ }^{a}$ CCAST (World Laboratory), P.O.Box 8730, Beijing 100080, China \\ ${ }^{c}$ Institute of Theoretical Physics, Academia Sinica, Beijing 100080, China \\ ${ }^{d}$ Graduate School of Science, Hiroshima University, Hiroshima 937-6256, Japan
}

(October 30, 2018)

\begin{abstract}
In the Minimal Supersymmetric Model (MSSM) the hitherto unconstrained flavor mixing between top-squark and charm-squark will induce the flavor-changing neutral-current (FCNC) interaction between top quark and charm quark, which then lead to various processes at the next generation linear collider (NLC), i.e., the top-charm associated productions via $e^{+} e^{-}, e^{-} \gamma$ and $\gamma \gamma$ collisions as well as the top quark rare decays $t \rightarrow c V(V=g, \gamma$ or $Z)$. All these processes involve the same part of the parameter space of the MSSM. Through a comparative analysis for all these processes at the NLC, we found that the best channel to probe such SUSY-induced top quark FCNC is the top-charm associated production in $\gamma \gamma$ collision, which occurs at a much higher rate than $e^{+} e^{-}$or $e^{-} \gamma$ collision and may reach the detectable level for some part of the parameter space. Since the rates predicted by the Standard Model are far below the detectable level, the observation of such FCNC events would be a robust evidence of new physics.
\end{abstract}

14.65.Ha 12.60.Jv 11.30.Pb

\section{INTRODUCTION}

The study of flavor-changing neutral-current (FCNC) interactions has been playing an important role in testing the Standard Model (SM) and probing new physics beyond the SM. As the most massive fermion in the SM, the top quark is naturally regarded to be more sensitive to new physics than other fermions. In the SM, due to the GIM mechanism, the top quark FCNC interactions are absent at tree level and extremely small at loop levels. In some new physics models beyond the SM the top quark FCNC may be significantly enhanced. Searching for the top quark FCNC would be a good probe for new physics.

Intensive activities to explore the top quark FCNC couplings have been undertaken in recent years. On the experimental side, the CDF and D0 collaborations have reported interesting bounds on the FCNC top quark decays [1] from Run 1 experiment and will tighten the bounds from the on-going Run 2 experiment. On the theoretical side, various FCNC top quark decays and top-charm associated productions at high energy colliders were extensively studied in the SM [2,3], the Minimal Supersymmetric Model (MSSM) [4-7] and other new physics models [8]. These studies showed that the SM predictions for such top quark FCNC processes are far below the detectable level and the MSSM can enhance them by several orders to make them potentially accessible at future collider experiments [9].

Due to its rather clean environment, the next generation linear collider (NLC) will be an ideal machine to probe new physics. In such a collider, in addition to $e^{+} e^{-}$collision, we can also realize $\gamma \gamma$ collision and $e^{-} \gamma$ collision with the photon beams generated by the backward Compton scattering of incident electron- and laser-beams [10]. The SUSY induced top-charm FCNC will give rise to various processes at the NLC, i.e., the top-charm associated productions via $e^{+} e^{-}, e^{-} \gamma$ and $\gamma \gamma$ collisions as well as the top quark rare decays $t \rightarrow c V(V=g, \gamma$ or $Z)$. It is noticable that some of these processes, like the top-charm associated productions in $\gamma \gamma$ or $e^{-} \gamma$ collision, have not been studied in the framework of the MSSM. The production in $\gamma \gamma$ collision may be more important than in $e^{+} e^{-}$collision studied in the literature [7]. The reason is twofold. Firstly, the process $\gamma \gamma \rightarrow t \bar{c}$ is a good probe of new physics because it is essentially free of any SM irreducible background [11]. Secondly, unlike the process $e^{+} e^{-} \rightarrow t \bar{c}$, which is $s$-channel suppressed in high energy collisions, there are $t$ - and $u$-channel contributions to $\gamma \gamma \rightarrow t \bar{c}$ and thus its cross section may be much larger at the NLC. It is also noticable that all these FCNC processes at the NLC involve the same part of the parameter space of the MSSM ${ }^{1}$. Therefore, it is necessary to perform a comparative analysis for all these processes to find out which process is best to probe the top quark FCNC. This is the aim of this article.

This paper is organized as follows. In Sec. II, we discuss the mixing between top-squark and charm-squark and derive the FCNC interaction Lagrangian in SUSY-QCD. In Sec. III, we calculate the $t \bar{c}$ productions in $\gamma \gamma$ and $e^{-} \gamma$

\footnotetext{
${ }^{1}$ Since the FCNC decay $t \rightarrow c h$ involves more parameters, we do not include it in our analysis.
} 
collisions induced by such FCNC SUSY-QCD interactions. Numerical results for these productions at the NLC are given in Sec. IV, with the comparison to $t \bar{c}$ production in $e^{+} e^{-}$collision and various FCNC top quark decays. Finally, a brief discussion and conclusion can be found in Sec. V.

\section{FLAVOR MIXING OF TOP-SQUARK AND CHARM-SQUARK AND THE INDUCED FCNC}

Many popular SUSY models predict the flavor mixings of sfermions. For the squark sector, despite of the possible strong constraints on the down type squark flavor mixings from the low-energy experimental data ${ }^{2}$, the mixings between top-squark and charm-squark are subject to no strong low-energy constraints [13].

Such a mixing between top-squark and charm-squark is well motivated in low-energy supergravity models (SUGRA) [14]. In these models squark mass matrices are diagonalized simultaneously as with the quark matrices at the Planck scale. But when the terms evolve down to low energy, this diagonality is violated by radiative corrections. Due to large top quark mass, at low energy it is found [15] that the mixing between top-squark and charm-squark may be significant. Note that only $\tilde{c}_{L}$ mixes with top-squark while $\tilde{c}_{R}$ does not in the approximation of neglecting charm quark mass. As shown in [15], the mixing between $\tilde{c}_{L}$ and $\tilde{t}_{L}$ is most likely to be large, which is proportional to a sum of some soft masses.

Motivated by the above arguments, in our analysis we assume the existence of the mixing between $\tilde{c}_{L}$ and $\tilde{t}_{L}$ and parameterize the mixing as $\delta_{L} M_{\tilde{Q}} M_{\tilde{Q}_{1}}$, where $M_{\tilde{Q}}\left(M_{\tilde{Q}_{1}}\right)$ is the soft mass parameter for left-handed squark of third (second) generation and $\delta_{L}$ is a dimensionless parameter representing the mixing strength. $\delta_{L}$ can be calculated in terms of other parameters in a given model like mSUGRA [15]. But in our calculation we retain it as a free parameter in the range of $0 \sim 1[13]$.

Considering the mixing between $\tilde{t}_{L}$ and $\tilde{t}_{R}$ and neglecting the mixing between $\tilde{c}_{L}$ and $\tilde{c}_{R}$, we obtain the $3 \times 3$ mass-square matrix in the basis $\left(\tilde{t}_{L}, \tilde{t}_{R}, \tilde{c}_{L}\right)$ :

$$
\mathcal{L}_{\text {mass }}=\left(\begin{array}{ccc}
\tilde{t}_{L}^{*} & \tilde{t}_{R}^{*} & \tilde{c}_{L}^{*}
\end{array}\right)\left(\begin{array}{ccc}
M_{\tilde{Q}}^{2}+m_{t}^{2}+D_{L} & m_{t} X & \delta_{L} M_{\tilde{Q}} M_{\tilde{Q}_{1}} \\
m_{t} X & M_{\tilde{U}}^{2}+m_{t}^{2}+D_{R} & 0 \\
\delta_{L} M_{\tilde{Q}} M_{\tilde{Q}_{1}} & 0 & M_{\tilde{Q}_{1}}^{2}+m_{c}^{2}+D_{L}
\end{array}\right)\left(\begin{array}{c}
\tilde{t}_{L} \\
\tilde{t}_{R} \\
\tilde{c}_{L}
\end{array}\right)
$$

where $D_{L} \equiv m_{Z}^{2} \cos (2 \beta)\left(\frac{1}{2}-\frac{2}{3} s_{W}^{2}\right), D_{R}=\frac{2}{3} m_{Z}^{2} \cos (2 \beta) s_{W}^{2}, X=A_{t}+\mu \cot \beta$ and $M_{\tilde{U}}^{2}$ is the soft-breaking mass term for right-handed top-squark. $A_{t}$ is the coefficient of the trilinear term $H_{2} \tilde{Q} \tilde{U}$ in soft-breaking terms, $\mu$ is the mixing mass parameter between $H_{1}$ and $H_{2}$ in the superpotential and $\tan \beta=v_{2} / v_{1}$ is ratio of the vacuum expectation values of the two Higgs doublets.

The mass-square matrix in Eq.(1) can be diagonalized by a $3 \times 3$ unitary matrix $V$, which rotates the interaction eigenstates $\left(\tilde{t}_{L}, \tilde{t}_{R}, \tilde{c}_{L}\right)$ into mass eigenstates $\tilde{q}_{1,2,3}$. Such a rotation results in the FCNC in both the weak interaction sector and the strong SUSY-QCD sector. Of course, the latter will be dominant. So in our analysis we only consider the FCNC in SUSY-QCD sector, given by

$$
\mathcal{L}_{F C N C}=-\sqrt{2} g_{s} T^{a}\left[\overline{\tilde{g}}_{a}\left(V_{i 1}^{\dagger} P_{L}-V_{i 2}^{\dagger} P_{R}\right) t \tilde{q}_{i}^{*}+\overline{\tilde{g}}_{a} V_{i 3}^{\dagger} P_{L} c \tilde{q}_{i}^{*}\right]
$$

where $P_{L, R}=\left(1 \mp \gamma_{5}\right) / 2$.

\section{CALCULATIONS}

The SUSY-QCD FCNC in Eq.(2) will induce the top-charm associated production in $\gamma \gamma$ collision, as shown in Fig. 1. Throughout our calculations the charge conjugate production channel, i.e., the production of $\bar{t} c$, has also been included. Neglecting the charm quark mass, the amplitude of this process is given by

$$
\mathcal{M}=2 i \alpha \alpha_{s} Q_{c}^{2} C_{F} \bar{u}_{t} \Gamma^{\mu \nu} P_{L} v_{c} \epsilon_{\mu}\left(\lambda_{1}\right) \epsilon_{\nu}\left(\lambda_{2}\right)
$$

with $Q_{c}=2 / 3, C_{F}=4 / 3$ and

\footnotetext{
${ }^{2}$ The down type squark flavor mixings in large $\tan \beta$ limit could enhance the FCNC $B$ decays by several orders [12] and will face tests in the on-going $B$-factory experiments.
} 


$$
\begin{aligned}
\Gamma^{\mu \nu}= & c_{1} p_{t}^{\mu} p_{t}^{\nu}+c_{2} p_{c}^{\mu} p_{c}^{\nu}+c_{3} p_{t}^{\mu} p_{c}^{\nu}+c_{4} p_{t}^{\nu} p_{c}^{\mu}+c_{5} p_{t}^{\mu} \gamma^{\nu}+c_{6} p_{c}^{\mu} \gamma^{\nu}+c_{7} p_{c}^{\nu} \gamma^{\mu}+c_{8} p_{t}^{\nu} \gamma^{\mu} \\
& +c_{9} g^{\mu \nu}+c_{10} \gamma^{\mu} \gamma^{\nu}+c_{11} p_{t}^{\mu} p_{t}^{\nu} k_{2}+c_{12} p_{c}^{\mu} p_{c}^{\nu} k_{2}+c_{13} p_{t}^{\mu} p_{c}^{\nu} k_{2}+c_{14} p_{t}^{\nu} p_{c}^{\mu} k_{2}+c_{15} p_{t}^{\mu} \gamma^{\nu} k_{2} \\
& +c_{16} p_{c}^{\mu} \gamma^{\nu} k_{2}+c_{17} p_{c}^{\nu} \gamma^{\mu} k_{2}+c_{18} p_{t}^{\nu} \gamma^{\mu} k_{2}+c_{19} g^{\mu \nu} k_{2}+c_{20} i \varepsilon^{\mu \nu \alpha \beta} \gamma_{\alpha} k_{2 \beta} .
\end{aligned}
$$

Here $k_{1,2}$ denote the momentum of incoming photons and $p_{t, c}$ the momentum of outgoing top and charm quarks. The coefficients $c_{i}$ can be obtained by a straightforward calculation of the diagrams shown in Fig. 1 and their expressions are presented in the Appendix.
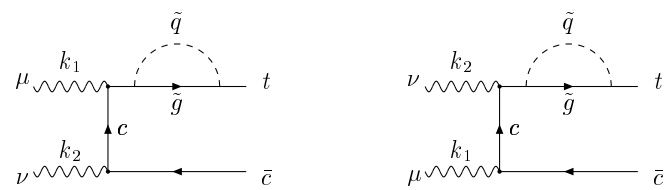

(a)
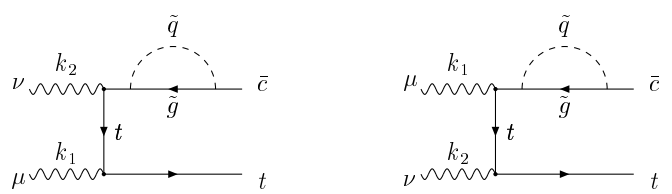

(b)
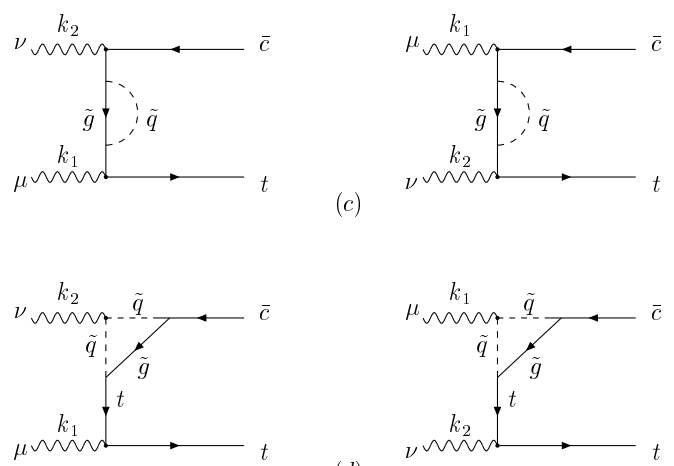

$(c)$
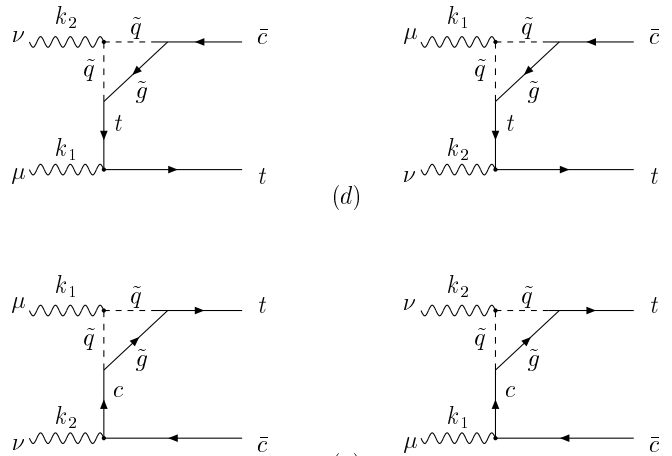

$(e)$
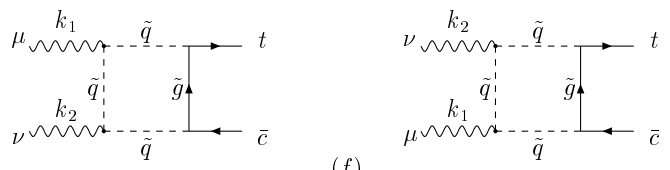

FIG. 1. Feynman diagrams contributing to the process $\gamma \gamma \rightarrow t \bar{c}$ in SUSY-QCD.

We checked that our results satisfy the Ward identity, $k_{1}^{\mu} \Gamma_{\mu \nu}=0$ and $k_{2}^{\nu} \Gamma_{\mu \nu}=0$. We also checked that all ultraviolet divergences canceled in our results due to the unitary of $V$, which is essentially guaranteed by the renormalizability of the MSSM. Note that when $t$ or $u$ approaches 0 , which is physically permitted, $c_{i}$ tend to be very large. Indeed, this is the advantage of such a process over $e^{+} e^{-} \rightarrow t \bar{c}$ which has only $s$-channel contribution and thus is suppressed at high energy colliders. 
The $t \bar{c}$ production in $e^{-} \gamma$ collision proceeds through the process $e^{-} \gamma \rightarrow e^{-} \gamma^{*} \gamma \rightarrow e^{-} t \bar{c}$, where the $\gamma$-beam is generated by the backward Compton scattering of incident electron- and laser-beam and the $\gamma^{*}$ is radiated from $e^{-}$ beam. The subprocess $\gamma^{*} \gamma \rightarrow t \bar{c}$ has the same Feynman diagrams shown in Fig. 1. In our calculation we use the Weizsäcker-Williams approximation [16] which treats $\gamma^{*}$ from $e^{-}$beam as a real photon. Thus the process can be approximated by the simpler fusion reaction $\gamma \gamma \rightarrow t \bar{c}$ and its cross section is given by

$$
\hat{\sigma}_{e \gamma \rightarrow e t \bar{c}}\left(s_{e \gamma}\right)=\int_{\left(m_{t}+m_{c}\right)^{2} / s_{e \gamma}}^{1} \mathrm{~d} x P_{\gamma / e}\left(x, E_{e}\right) \hat{\sigma}_{\gamma \gamma \rightarrow t \bar{c}}\left(s_{\gamma \gamma}=x s_{e \gamma}\right)
$$

where $P_{\gamma / e}\left(x, E_{e}\right)$ is the probability of finding a photon with a fraction $x$ of energy $E_{e}$ in an ultrarelativistic electron and is given by [16]

$$
P_{\gamma / e}\left(x, E_{e}\right)=\frac{\alpha}{\pi}\left(\frac{1+(1-x)^{2}}{x}\left(\ln \frac{E_{e}}{m_{e}}-\frac{1}{2}\right)+\frac{x}{2}\left(\ln \left(\frac{2}{x}-2\right)+1\right)+\frac{(2-x)^{2}}{2 x} \ln \left(\frac{2-2 x}{2-x}\right)\right) .
$$

Note that there are also intermediate $Z$-boson contribution for the process $e \gamma \rightarrow$ et $\bar{c}$. However, such contributions are suppressed by the probability function of finding a $Z$-boson in an ultrarelativistic electron [17] and can be safely neglected.

For both $\gamma \gamma$ collider and $e \gamma$ collider, the photon beams are generated by the backward Compton scattering of incident electron- and laser-beams just before the interaction point. The events number is obtained by convoluting the cross section with the photon beam luminosity distribution. For $\gamma \gamma$ collider the events number is obtained by

$$
N_{\gamma \gamma \rightarrow t \bar{c}}=\int \mathrm{d} \sqrt{s_{\gamma \gamma}} \frac{\mathrm{d} \mathcal{L}_{\gamma \gamma}}{\mathrm{d} \sqrt{s_{\gamma \gamma}}} \hat{\sigma}_{\gamma \gamma \rightarrow t \bar{c}}\left(s_{\gamma \gamma}\right) \equiv \mathcal{L}_{e e} \sigma_{\gamma \gamma \rightarrow t \bar{c}}\left(s_{e e}\right),
$$

where $\mathrm{d} \mathcal{L}_{\gamma \gamma} / \mathrm{d} \sqrt{s_{\gamma \gamma}}$ is the photon beam luminosity distribution and $\sigma_{\gamma \gamma \rightarrow t \bar{c}}\left(s_{e e}\right)$, with $s_{e e}$ being the energy-square of $e^{+} e^{-}$collison, is defined as the effective cross section of $\gamma \gamma \rightarrow t \bar{c}$. In optimum case, it can be written as [18]

$$
\sigma_{\gamma \gamma \rightarrow t \bar{c}}\left(s_{e e}\right)=\int_{\left(m_{t}+m_{c}\right) / \sqrt{s_{e e}}}^{x_{\max }} 2 z \mathrm{~d} z \hat{\sigma}_{\gamma \gamma \rightarrow t \bar{c}}\left(s_{\gamma \gamma}=z^{2} s_{e e}\right) \int_{z^{2} / x_{\max }}^{x_{\max }} \frac{\mathrm{d} x}{x} F_{\gamma / e}(x) F_{\gamma / e}\left(\frac{z^{2}}{x}\right),
$$

where $F_{\gamma / e}$ denotes the energy spectrum of the back-scattered photon for unpolarized initial electrons and laser photon beams given by

$$
F_{\gamma / e}(x)=\frac{1}{D(\xi)}\left(1-x+\frac{1}{1-x}-\frac{4 x}{\xi(1-x)}+\frac{4 x^{2}}{\xi^{2}(1-x)^{2}}\right) .
$$

The definitions of parameters $\xi, D(\xi)$ and $x_{\max }$ can be found in [18]. In our numerical calculation, we choose $\xi=4.8$, $D(\xi)=1.83$ and $x_{\max }=0.83$.

For the $e^{-} \gamma$ collider the effective cross section of $e \gamma \rightarrow$ et $\bar{c}$ is defined as

$$
\begin{aligned}
\sigma_{e \gamma \rightarrow e t \bar{c}}\left(s_{e e}\right) & =\frac{1}{\mathcal{L}_{e e}} \int \mathrm{d} \sqrt{s_{e \gamma}} \frac{\mathrm{d} \mathcal{L}_{e \gamma}}{\mathrm{d} \sqrt{s_{e \gamma}}} \hat{\sigma}_{e \gamma \rightarrow e t \bar{c}}\left(s_{e \gamma}\right) \\
& =\int_{\left(m_{t}+m_{c}\right) / \sqrt{s_{e e}}}^{x_{\max }} 2 z \mathrm{~d} z \hat{\sigma}_{\gamma \gamma \rightarrow t \bar{c}}\left(s_{\gamma \gamma}=z^{2} s_{e e}\right) \int_{z^{2} / x_{\max }}^{1} \frac{\mathrm{d} x}{x} P_{\gamma / e}\left(x, E_{e}\right) F_{\gamma / e}\left(\frac{z^{2}}{x}\right) .
\end{aligned}
$$

The process $e^{+} e^{-} \rightarrow t \bar{c}$ and the top quark rare decays $t \rightarrow c V(V=\gamma, Z, g)$ were already calculated in the literature. Here for comparison we recalculated all of them. The lengthy expressions are not presented here.

\section{NUMERICAL RESULTS}

The relevant SUSY parameters all the top-charm productions and top quark rare decays are $\delta_{L}, X\left(=A_{t}+\mu \cot \beta\right)$, $M_{\tilde{g}}, M_{\tilde{Q}}, M_{\tilde{U}}, M_{\tilde{Q} 1}$ and $\tan \beta$. To find out typical magnitudes of these processes, we scan over these SUSY parameters by requiring $5 \leq \tan \beta \leq 50, m_{\tilde{q}} \geq 86.4 \mathrm{GeV}[19], m_{\tilde{g}}>190 \mathrm{GeV}[19], m_{\tilde{Q} 1} \simeq 1 \mathrm{TeV}$ and restricting other soft mass parameters to be of sub-TeV scale.

Our scan results are plotted in Figs. 2 and 3. For the productions one sees that the typical values are $\sigma(\gamma \gamma \rightarrow$ $t \bar{c}) \sim 10^{-2} \mathrm{fb}$ and $\sigma(e \gamma \rightarrow e t \bar{c}) \sim 10^{-3} \mathrm{fb}$ and in optimum cases $\sigma(\gamma \gamma \rightarrow t \bar{c}) \simeq 0.7 \mathrm{fb}$ and $\sigma(e \gamma \rightarrow e t \bar{c}) \simeq 0.04 \mathrm{fb}$. While for the process $e^{+} e^{-} \rightarrow t \bar{c}$, the cross section can only reach $0.02 \mathrm{fb}$, one order of magnitude lower than that of $\gamma \gamma \rightarrow t \bar{c}$ and comparable with that of $e \gamma \rightarrow e t \bar{c}$. The reason for this, as we pointed out before, is due to the $t$-channel enhancement for $\gamma \gamma \rightarrow t \bar{c}$ and $s$-channel suppression for $e^{+} e^{-} \rightarrow t \bar{c}$. For the top quark rare decays we see that the optimum values are $\operatorname{Br}(t \rightarrow c g) \sim 10^{-5}, \operatorname{Br}(t \rightarrow c Z) \sim 10^{-7}$ and $\operatorname{Br}(t \rightarrow c \gamma) \sim 10^{-7}$, which agree with previous results [5]. 


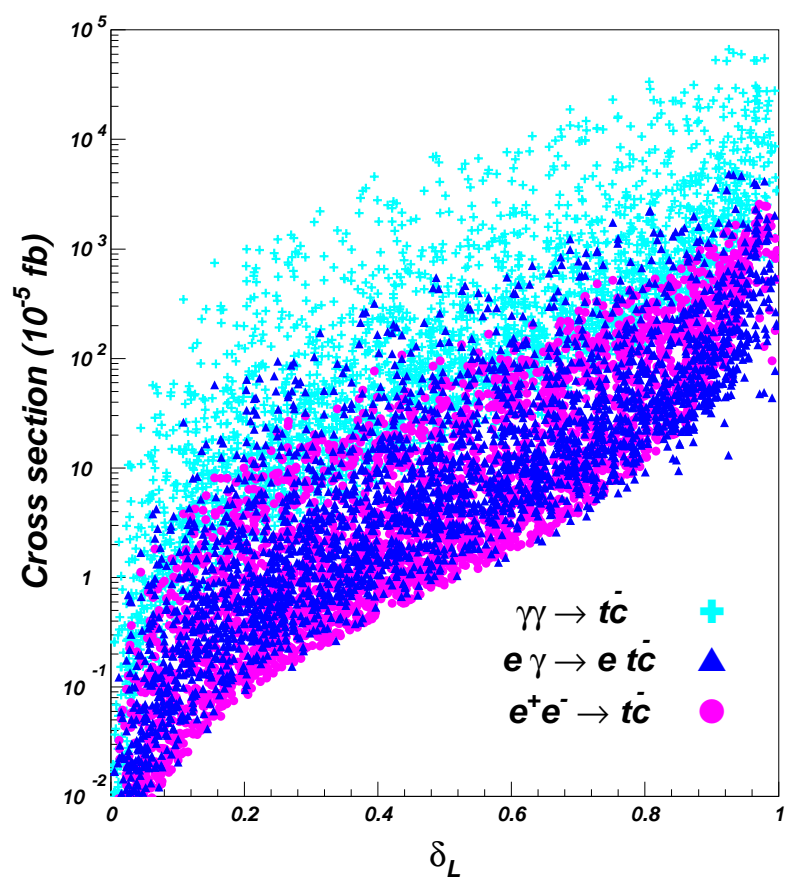

FIG. 2. The scattered plot of SUSY-QCD contribution to processes $\gamma \gamma \rightarrow t \bar{c}, e \gamma \rightarrow e t \bar{c}$ and $e^{+} e^{-} \rightarrow t \bar{c}$ for $\sqrt{s_{e e}}=500 \mathrm{GeV}$.

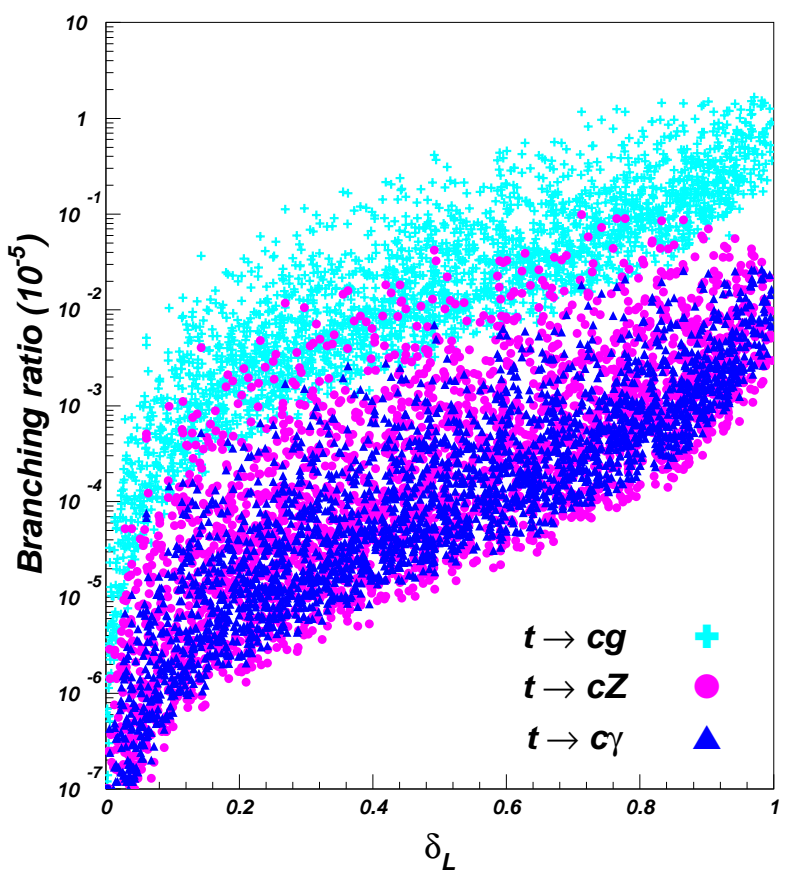

FIG. 3. Same as Fig. 2, but for FCNC top quark rare decays.

Comparing with predictions in other models, such as R-parity violating model [20] and the type III two Higgs doublet model [21], we find that the optimum value of $\sigma(\gamma \gamma \rightarrow t \bar{c})$ in SUSY-QCD is larger. Note that our results for $\sigma\left(e^{+} e^{-} \rightarrow t \bar{c}\right)$ are quite different from those in Ref. [7] where the optimum value of $\sigma\left(e^{+} e^{-} \rightarrow t \bar{c}\right)$ can reach $0.1 \mathrm{fb}$. The reason for the difference is that in Ref. [7] a non-unitary flavor changing matrix is used. During our scan, we find that, only in case of a light top-squark with mass of $100 \sim 200 \mathrm{GeV}$ and a large mass splitting of a few hundreds $\mathrm{GeV}$ in squark spectrum can relative large cross sections of these processes be obtained. This is due to a GIM-like 
cancellation which occurs in the limit of degenerate squark masses as a result of the unitarity of the rotation matrix $V$.

The SM and MSSM predictions for the top-charm FCNC processes are summarized in Table I. Note that since the SM prediction for the process $\gamma \gamma \rightarrow t \bar{c}$ has not been done in the literature, the SM value shown in the table is calculated by us. This is an arduous work. There are 66 diagrams contributing $\gamma \gamma \rightarrow t \bar{c}$ in $R_{\xi}$ gauge and often the contribution from a single box diagram can be decomposed into dozens of terms. In order to avoid artificial error, we use FormCalc [22] in our calculations. From Table I, we see that the MSSM can enhance the cross section of top-charm associated production processes by several orders.

Table 1: Theoretical predictions for the top-quark FCNC processes. SUSY-QCD predictions are the maximum values. The collider energy is $500 \mathrm{GeV}$ for productions.

\begin{tabular}{|l|l|l|}
\hline & SM & SUSY QCD \\
\hline$\sigma(\gamma \gamma \rightarrow t \bar{c})$ & $\mathcal{O}\left(10^{-8}\right) \mathrm{fb}$ & $\mathcal{O}\left(10^{-1}\right) \mathrm{fb}$ \\
\hline$\sigma\left(e^{-} \gamma \rightarrow e^{-} t \bar{c}\right)$ & $\mathcal{O}\left(10^{-9}\right) \mathrm{fb}$ & $\mathcal{O}\left(10^{-2}\right) \mathrm{fb}$ \\
\hline$\sigma\left(e^{+} e^{-} \rightarrow t \bar{c}\right)$ & $\mathcal{O}\left(10^{-10}\right) \mathrm{fb}$ & $\mathcal{O}\left(10^{-2}\right) \mathrm{fb}$ \\
\hline $\mathcal{B} r(t \rightarrow c g)$ & $\mathcal{O}\left(10^{-11}\right)$ & $\mathcal{O}\left(10^{-5}\right)$ \\
\hline $\mathcal{B} r(t \rightarrow c Z)$ & $\mathcal{O}\left(10^{-13}\right)$ & $\mathcal{O}\left(10^{-7}\right)$ \\
\hline $\mathcal{B} r(t \rightarrow c \gamma)$ & $\mathcal{O}\left(10^{-13}\right)$ & $\mathcal{O}\left(10^{-7}\right)$ \\
\hline
\end{tabular}

The behaviours of the SM cross sections versus the collider energy are shown in Fig. 4 for three different production processes. We see that the cross section of $e^{+} e^{-} \rightarrow t \bar{c}$ drops quickly with the increase of collider energy due to the $s$-channel suppression.

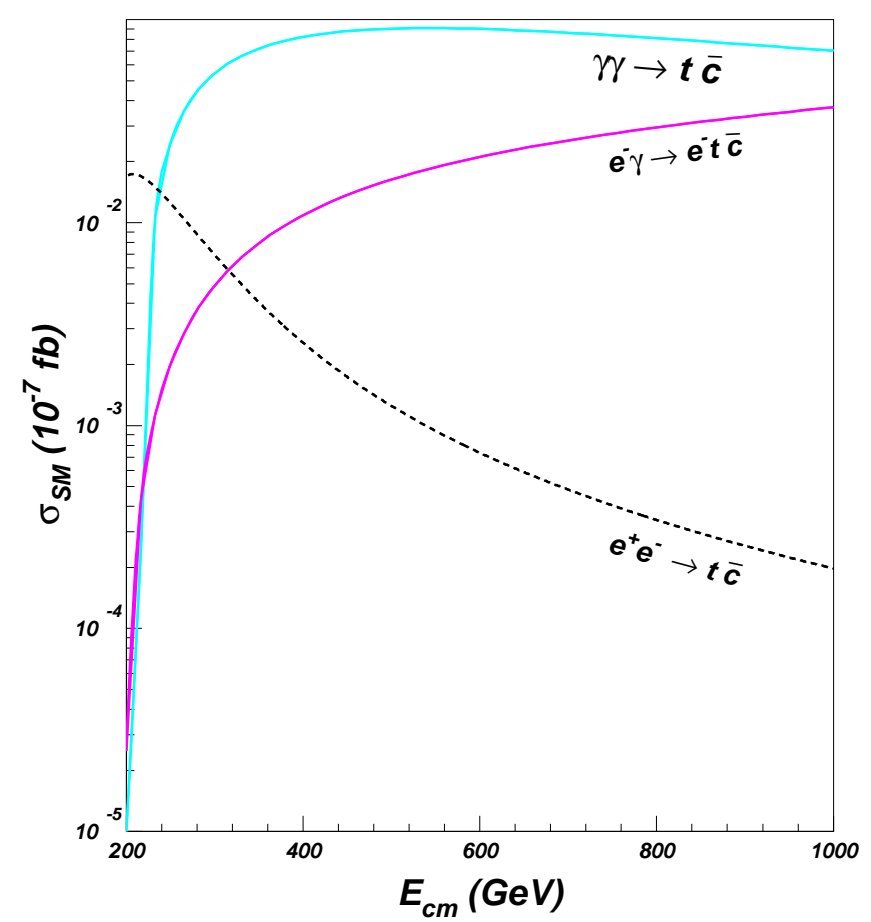

FIG. 4. Cross sections of top-charm associated production processes versus collider energy $E_{c m}=\sqrt{s_{e e}}$ in the SM.

Let us focus on the most important process $\gamma \gamma \rightarrow t \bar{c}$ and study some of its features. For example we fix $\tan \beta=30$ and $M_{\tilde{Q} 1}=1000 \mathrm{GeV}$, and choose $M_{\tilde{Q}}=600 \mathrm{GeV}, M_{\tilde{U}}=400 \mathrm{GeV}$ for scenario I (relatively light squarks for the third-family) and $M_{\tilde{Q}}=800 \mathrm{GeV}, M_{\tilde{U}}=600 \mathrm{GeV}$ for scenario II (relatively heavy squarks for the third-family). 


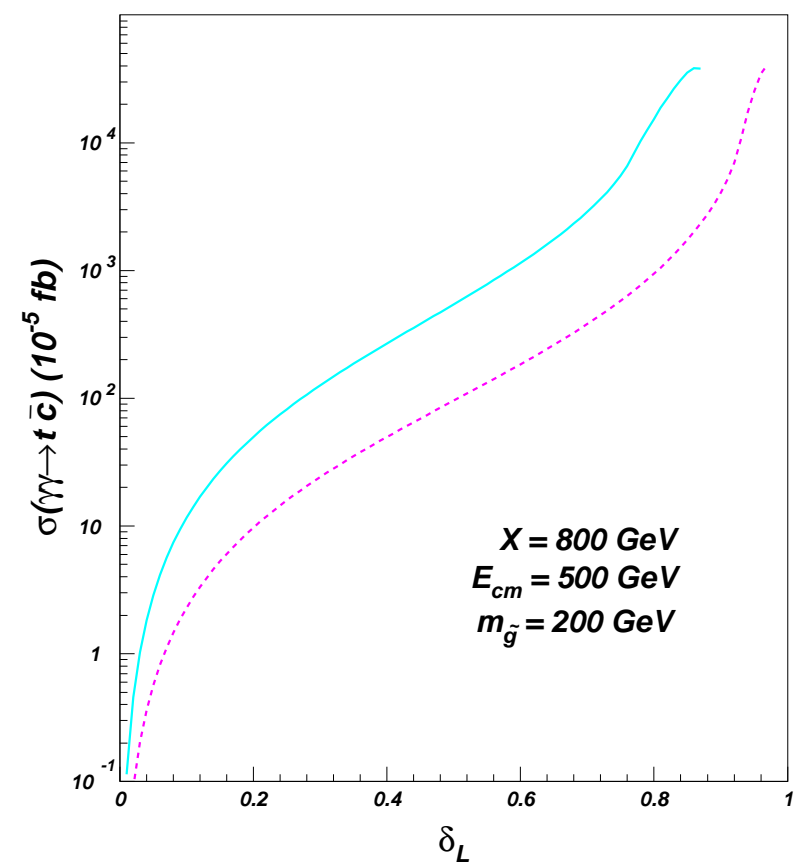

FIG. 5. The SUSY-QCD correction to $\sigma_{\gamma \gamma \rightarrow t \bar{c}}$ as a function of $\delta_{L}$. The solid curve corresponds to scenario I (relatively light squarks for the third-family) and the dashed curve corresponds to scenario II (relatively heavy squarks for the third-family).

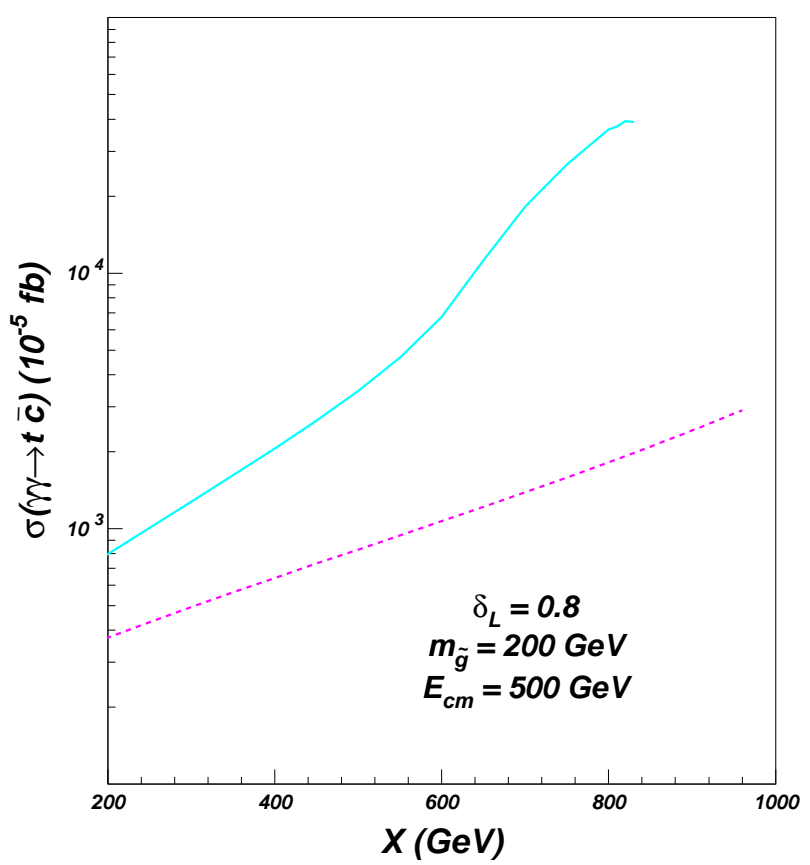

FIG. 6. Same as Fig 5, but for $\sigma(\gamma \gamma \rightarrow t \bar{c})$ as a function of $X\left(=A_{t}+\mu \cot \beta\right)$.

The cross section of $\gamma \gamma \rightarrow t \bar{c}$ as a function of $\delta_{L}$ is shown in Fig. 5. This figure shows that the cross section is enhanced dramatically with the increase of $\delta_{L}$. Clearly this is due to the fact that large $\delta_{L}$ will not only enhance the mixing between top-squark and charm-squark but also enlarge the mass splitting between the squarks. In Fig. 6 we show the dependence of $\sigma(\gamma \gamma \rightarrow t \bar{c})$ on $X$. We see that $\sigma(\gamma \gamma \rightarrow t \bar{c})$ increases as $X$ gets large. The reason is that large $X$ enhances the mass splitting between the top-squarks and thus lead to a weak cancellation between different Feynman diagrams. 


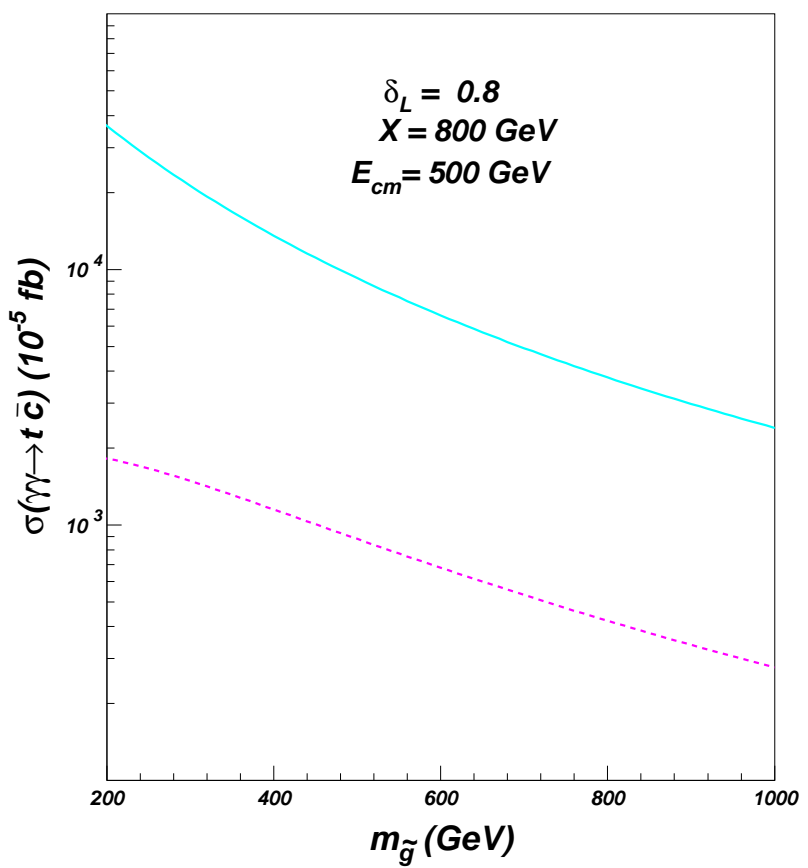

FIG. 7. Same as Fig 5, but for the dependence on luino mass.

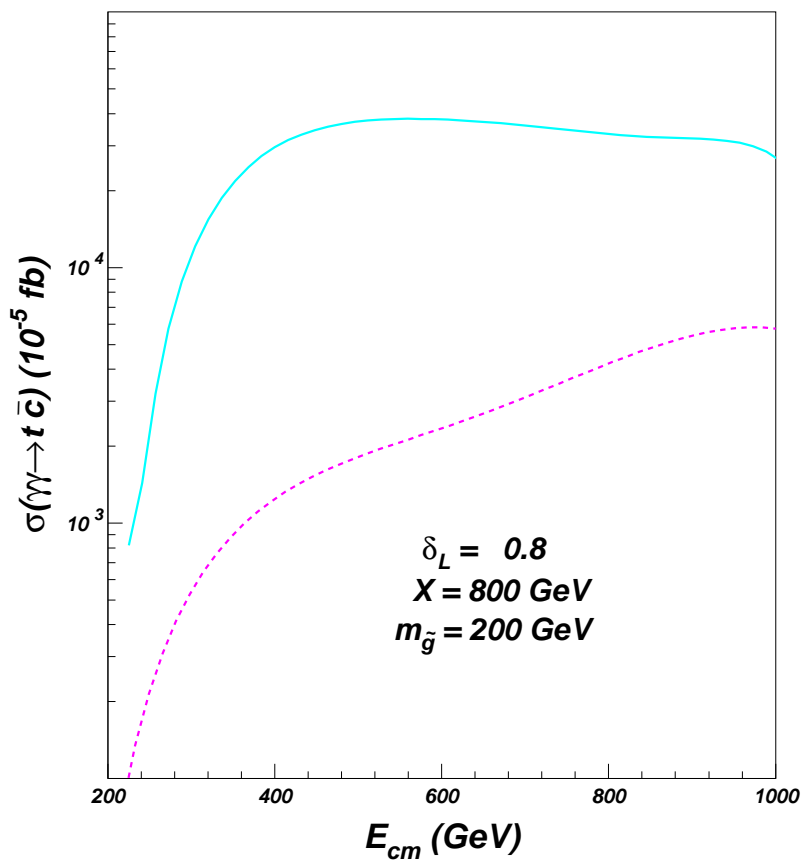

FIG. 8. Same as Fig 5, but for the dependence on $\sqrt{s}$.

The dependence of $\sigma(\gamma \gamma \rightarrow t \bar{c})$ on $m_{\tilde{g}}$ and $\sqrt{s}$ is shown in Fig. 7 and Fig. 8, respectively. Fig. 7 shows that the cross section drops when gluino becomes heavier, showing the decoupling effect of the MSSM.

From Figs. 5-8 one finds that the cross section in scenario I is always larger than that in scenario II. The reason is that scenario I has the relative light squarks for the third-family which lead to a larger mass splitting in squark spectrum. 


\section{SUMMARY AND CONCLUSION}

We have performed a comparative analysis for the SUSY-induced top-charm FCNC processes at the NLC. These processes include the top-charm associated productions via $e^{+} e^{-}, e^{-} \gamma$ and $\gamma \gamma$ collisions as well as the top quark rare decays $t \rightarrow c V(V=g, \gamma$ or $Z)$, all of which involve the same SUSY parameters.

In the production channels the $t \bar{c}$ production in $\gamma \gamma$ collision was found to occur at a much higher rate than $e^{+} e^{-}$ or $e^{-} \gamma$ collision. In some part of parameter space, the production rate of $\gamma \gamma \rightarrow t \bar{c}$ can reach $0.7 \mathrm{fb}$. This means we may have 70 events each year for the designed luminosity of $100 \mathrm{fb}^{-1} /$ year at the NLC. Since the SM value of the production rate is completely negligible, the observation of such $t \bar{c}$ events would be a robust indirect evidence of SUSY.

Note that in practical experimental searches of such $t \bar{c}$ events, a careful study of backgrounds is needed. To efficiently suppress the backgrounds, the signal events may be hurt by $50 \%$ or more $[11,23]$. Using polarization at a linear collider can help to suppress the background, as analyzed in the second reference of [9].

In the rare decay channels the $t \rightarrow c g$ was found to have the largest branching ratio which can reach $10^{-5}$, in agreement with previous studies. Although $10^{5} t \bar{t}$ events could be produced at the NLC each year, studies [24] showed that the sensitivity to such rare decays can only reach $5 \times 10^{-4}$. So the MSSM prediction for the branching ratio of $10^{-5}$ is too low to be accessible at the NLC unless the designed luminosity can be further upgraded.

Therefore, we conclude that, to probe the SUSY-induced FCNC top quark interactions at the NLC, $t \bar{c}$ production in $\gamma \gamma$ collision is the best channel.

Note that in our analysis we assumed flavor mixing occurs between $\tilde{c}_{L}$ and $\tilde{t}_{L}$, which is favored in SUGRA models. Other kinds of mixings like the mixing between $\tilde{c}_{R}$ and $\tilde{t}_{L}[25,26]$ may also be of phenomenological interest. However, we believe our conclusion will be quite model-independent since our results reflect the basic features of the processes.

\section{ACKNOWLEDGMENT}

We thank Kaoru Hagiwara and Tao Han for very helpful discussions. This work is supported by Young Outstanding Foundation of Academia Sinica.

\section{APPENDIX}

Before presenting the explicit form of $\Gamma^{\mu \nu}$, we define the following abbreviations

$$
\begin{aligned}
\hat{s} & =\left(k_{1}+k_{2}\right)^{2}=\left(p_{t}+p_{c}\right)^{2}, \\
\hat{t} & =\left(p_{t}-k_{2}\right)^{2}=\left(k_{1}-p_{c}\right)^{2}, \\
\hat{u} & =\left(p_{t}-k_{1}\right)^{2}=\left(k_{2}-p_{c}\right)^{2}, \\
\hat{V}_{13} B_{i}^{a} & =\sum_{\beta=1}^{3} V_{1 \beta} V_{\beta 3}^{\dagger} B_{i}\left(-p_{t}, m_{\tilde{U}_{\beta}}, m_{\tilde{g}}\right), \\
\hat{V}_{13} B_{i}^{b} & =\sum_{\beta=1}^{3} V_{1 \beta} V_{\beta 3}^{\dagger} B_{i}\left(p_{c}, m_{\tilde{U}_{\beta}}, m_{\tilde{g}}\right), \\
\hat{V}_{13} B_{i}^{c}= & \sum_{\beta=1}^{3} V_{1 \beta} V_{\beta 3}^{\dagger} B_{i}\left(-p_{t}+k_{2}, m_{\tilde{U}_{\beta}}, m_{\tilde{g}}\right), \\
\hat{V}_{13} C_{i j}^{e}= & \sum_{\beta=1}^{3} V_{1 \beta} V_{\beta 3}^{\dagger} C_{i j}\left(k_{1}, p_{c}-k_{1}, m_{\tilde{U}_{\beta}}, m_{\tilde{U}_{\beta}}, m_{\tilde{g}}\right), \\
\hat{V}_{13} C_{i j}^{d}= & \sum_{\beta=1}^{3} V_{1 \beta} V_{\beta 3}^{\dagger} C_{i j}\left(k_{2},-p_{t}, m_{\tilde{U}_{\beta}}, m_{\tilde{U}_{\beta}}, m_{\tilde{g}}\right), \\
\hat{V}_{13} D_{i j}^{1}= & \sum_{\beta=1}^{3} V_{1 \beta} V_{\beta 3}^{\dagger} D_{i j}\left(k_{2},-p_{t},-p_{c}, m_{\tilde{U}_{\beta}}, m_{\tilde{U}_{\beta}}, m_{\tilde{g}}, m_{\tilde{U}_{\beta}}\right),
\end{aligned}
$$


where $V$ is squark mixing matrix and $\mathrm{B}, \mathrm{C}$ and $\mathrm{D}$ are loop functions defined in [27].

Then $\Gamma^{\mu \nu}$ is given by

$$
\Gamma^{\mu \nu}=\Gamma_{t}^{\mu \nu}+\Gamma_{u}^{\mu \nu}+\Gamma_{B o x 1}^{\mu \nu}+\Gamma_{B o x 2}^{\mu \nu}
$$

with $\left.\Gamma_{u}^{\mu \nu}=\left.\Gamma_{t}^{\mu \nu}\right|_{\left(k_{1} \leftrightarrow k_{2}, \mu \leftrightarrow \nu, t \leftrightarrow u\right.}\right)$ and $\left.\Gamma_{B o x 2}^{\mu \nu}=\left.\Gamma_{B o x 1}^{\mu \nu}\right|_{\left(k_{1} \leftrightarrow k_{2}, \mu \leftrightarrow \nu, t \leftrightarrow u\right.}\right) . \Gamma_{t}^{\mu \nu}$ and $\Gamma_{B o x 1}^{\mu \nu}$ in Eq.(20) can be expressed in the form of Eq.(4) and their corresponding coefficients $c_{i}$ are given as

$$
\begin{aligned}
& c_{t, 1}=\frac{4}{\hat{t}-m_{c}^{2}}\left(m_{t} \hat{V}_{13}\left(C_{22}^{d}-C_{23}^{d}\right)-m_{\tilde{g}} \hat{V}_{23} C_{12}^{d}\right), \\
& c_{t, 4}=\frac{-4}{\hat{t}-m_{t}^{2}}\left(m_{t} \hat{V}_{13}\left(C_{23}^{e}-C_{22}^{e}\right)+m_{\tilde{g}} \hat{V}_{23} C_{12}^{e}\right)+c_{t, 1}, \\
& c_{t, 5}=\frac{-1}{m_{t}^{2}\left(\hat{t}-m_{c}^{2}\right)}\left(m_{t}^{2} \hat{V}_{13}\left(B_{0}^{a}+B_{1}^{a}\right)-m_{\tilde{g}} m_{t} \hat{V}_{23} B_{0}^{a}\right), \\
& -\frac{1}{m_{t}^{2}\left(\hat{t}-m_{t}^{2}\right)} m_{\tilde{g}} m_{t} \hat{V}_{23} B_{0}^{b}-\frac{1}{\left(\hat{t}-m_{t}^{2}\right)\left(\hat{t}-m_{c}^{2}\right)}, \\
& \times\left(\hat{t} \hat{V}_{13}\left(B_{0}^{c}+B_{1}^{c}\right)-m_{\tilde{g}} m_{t} \hat{V}_{23} B_{0}^{c}\right) \\
& +\frac{2}{\hat{t}-m_{c}^{2}} \hat{V}_{13} C_{24}^{d}+\frac{2}{\hat{t}-m_{t}^{2}} \hat{V}_{13} C_{24}^{e}, \\
& c_{t, 6}=-2 \hat{V}_{13}\left(C_{23}^{e}-C_{22}^{e}\right)+c_{t, 5}, \\
& c_{t, 8}=\frac{2}{m_{t}^{2}\left(\hat{t}-m_{c}^{2}\right)}\left(m_{t}^{2} \hat{V}_{13}\left(B_{0}^{a}+B_{1}^{a}\right)-m_{\tilde{g}} m_{t} \hat{V}_{23} B_{0}^{a}\right) \\
& +\frac{2}{m_{t}^{2}\left(\hat{t}-m_{t}^{2}\right)} m_{\tilde{g}} m_{t} \hat{V}_{23} B_{0}^{b}+\frac{2}{\left(\hat{t}-m_{t}^{2}\right)\left(\hat{t}-m_{c}^{2}\right)} \\
& \times\left(\hat{t} \hat{V}_{13}\left(B_{0}^{c}+B_{1}^{c}\right)-m_{\tilde{g}} m_{t} \hat{V}_{23} B_{0}^{c}\right) \\
& -\frac{2}{\hat{t}-m_{c}^{2}}\left(\hat{t} \hat{V}_{13}\left(C_{12}^{d}+C_{23}^{d}\right)+m_{t}^{2} \hat{V}_{13}\left(C_{22}^{d}-C_{23}^{d}\right)\right. \\
& \left.+2 \hat{V}_{13} C_{24}^{d}-m_{\tilde{g}} m_{t} \hat{V}_{23} C_{12}^{d}\right)-\frac{4}{\hat{t}-m_{t}^{2}} \hat{V}_{13} C_{24}^{e}, \\
& c_{t, 10}=\frac{-m_{t}}{m_{t}^{2}\left(\hat{t}-m_{c}^{2}\right)}\left(m_{t}^{2} \hat{V}_{13}\left(B_{0}^{a}+B_{1}^{a}\right)-m_{\tilde{g}} m_{t} \hat{V}_{23} B_{0}^{a}\right) \\
& -\frac{m_{t}}{\left(\hat{t}-m_{t}^{2}\right)\left(\hat{t}-m_{c}^{2}\right)}\left(\hat{t} \hat{V}_{13}\left(B_{0}^{c}+B_{1}^{c}\right)-m_{\tilde{g}} m_{t} \hat{V}_{23} B_{0}^{c}\right) \\
& +\frac{2 m_{t}}{\hat{t}-m_{c}^{2}} \hat{V}_{13} C_{24}^{d}+\frac{\hat{t}}{\left(\hat{t}-m_{t}^{2}\right)\left(\hat{t}-m_{c}^{2}\right)}\left(m_{t} \hat{V}_{13}\left(B_{0}^{c}+B_{1}^{c}\right)-m_{\tilde{g}} \hat{V}_{23} B_{0}^{c}\right), \\
& c_{t, 16}=\frac{2}{\hat{t}-m_{t}^{2}}\left(m_{t} \hat{V}_{13}\left(C_{23}^{e}-C_{22}^{e}\right)+m_{\tilde{g}} \hat{V}_{23} C_{12}^{e}\right), \\
& c_{t, 18}=\frac{-2}{\hat{t}-m_{c}^{2}}\left(m_{t} \hat{V}_{13}\left(C_{22}^{d}-C_{23}^{d}\right)-m_{\tilde{g}} \hat{V}_{23} C_{12}^{d}\right), \\
& c_{t, 19}=-c_{t, 20}=-c_{t, 5}, \\
& c_{B o x 1,1}=4\left(m_{t} \hat{V}_{13}-m_{\tilde{g}} \hat{V}_{23}\right)\left(D_{22}-D_{24}\right)+4 m_{t} \hat{V}_{13}\left(D_{32}-D_{36}\right) \text {, } \\
& c_{B o x 1,2}=4\left(m_{t} \hat{V}_{13}-m_{\tilde{g}} \hat{V}_{23}\right)\left(D_{23}-D_{25}\right)-4 m_{t} \hat{V}_{13}\left(D_{310}-D_{39}\right) \text {, } \\
& c_{B o x 1,3}=-4\left(m_{t} \hat{V}_{13}-m_{\tilde{g}} \hat{V}_{23}\right)\left(D_{25}-D_{26}\right)-4 m_{t} \hat{V}_{13}\left(D_{310}-D_{37}\right) \text {, } \\
& c_{B o x 1,4}=-4\left(m_{t} \hat{V}_{13}-m_{\tilde{g}} \hat{V}_{23}\right)\left(D_{24}-D_{26}\right)-4 m_{t} \hat{V}_{13}\left(D_{36}-D_{37}\right) \text {, } \\
& c_{\text {Box } 1,5}=-4 \hat{V}_{13}\left(D_{311}-D_{312}\right) \text {, } \\
& c_{B o x 1,6}=-4 \hat{V}_{13}\left(D_{311}-D_{313}\right) \text {, } \\
& c_{B o x 1,7}=4 \hat{V}_{13} D_{313} \text {, } \\
& c_{\text {Box } 1,8}=4 \hat{V}_{13} D_{312}, \\
& c_{B o x 1,9}=-4\left(m_{t} \hat{V}_{13}-m_{\tilde{g}} \hat{V}_{23}\right) D_{27}+4 m_{t} \hat{V}_{13} D_{312} \text {, }
\end{aligned}
$$




$$
\begin{aligned}
& c_{B o x 1,11}=-4 \hat{V}_{13}\left(D_{22}-D_{24}-D_{34}+D_{36}\right), \\
& c_{B o x 1,12}=-4 \hat{V}_{13}\left(D_{23}-D_{25}-D_{35}+D_{38}\right), \\
& c_{B o x 1,13}=4 \hat{V}_{13}\left(D_{25}-D_{26}-D_{310}+D_{35}\right), \\
& c_{B o x 1,14}=4 \hat{V}_{13}\left(D_{24}-D_{26}-D_{310}+D_{34}\right), \\
& c_{B o x 1,19}=4 \hat{V}_{13}\left(D_{27}-D_{311}\right) .
\end{aligned}
$$

[1] M. Paulini, hep-ex/9701019; J. Incandela (CDF), FERMILAB-CONF-95/237-E(1995); D. Gerdes, hep-ex/9706001; T. J. Lecompte (CDF), FERMILAB-CONF-96/021-E (1996); A. P. Heinson (D0), hep-ex/9605010.

[2] For the FCNC top quark decays in the SM, see, G. Eilam, J. L. Hewett and A. Soni, Phys. Rev. D 44, 1473 (1991); B. Mele, S. Petrarca, A. Soddu, Phys. Lett. B 435, 401 (1998).

[3] C.-H. Chang, X.-Q. Li, J.-X. Wang, M.-Z. Yang, Phys. Lett. B 313, 389 (1993) C.-S. Huang, X.-H. Wu and S.-H. Zhu, Phys. Lett. B 452, 14(1999).

[4] For a review see, e.g., H. E. Haber and G. L. Kane, Phys. Rep.117, 75 (1985).

[5] For $t \rightarrow c V$ in the MSSM, see, C. S. Li, R. J. Oakes and J. M. Yang, Phys. Rev. D 49, 293 (1994); G. Couture, C. Hamzaoui and H. Konig, Phys. Rev. D 52, 1713 (1995); J. L. Lopez, D. V. Nanopoulos and R. Rangarajan, Phys. Rev. D 56, 3100 (1997); G. M. de Divitiis, R. Petronzio and L. Silvestrini, Nucl. Phys. B 504, 45 (1997). J. M. Yang, B.-L. Young and X. Zhang, Phys. Rev. D 58, 055001 (1998).

[6] For $t \rightarrow c h$ in the MSSM, see, J. M. Yang and C. S. Li, Phys. Rev. D 49, 3412 (1994); J. Guasch, and J. Sola, Nucl. Phys. B 562, 3 (1999); G. Eilam, A. Gemintern, T. Han, J.M. Yang, X. Zhang, Phys. Lett. B 510, 227 (2001).

[7] C. S. Li, X. Zhang and S. H. Zhu, Phys. Rev. D 60, 077702 (1999).

[8] J. L. Diaz-Cruz, et al. Phys. Rev. D 41, 891(1990); G. Eilam, J. L. Hewett and A. Soni, Phys. Rev. D 44, 1473 (1991); X.L. Wang et al., Phys. Rev. D 50, 5781 (1994); J. Phys. G20, L91 (1994); Commun. Theor. Phys. 24, 359 (1995); David Atwood, L. Reina and A. Soni, Phys. Rev. D 53, 1199 (1996); S. Bar-Shalom, et al., Phys. Rev. Lett. 79, 1217(1997); W. S. Hou, G.-L. Lin and C.-Y. Ma, Phys. Rev. D 56, 7434(1997); S. Bejar, J. Guasch and J. Sola, Nucl. Phys. B 600, 21 (2001); R. A. Diaz, R. Martinez, J.-A. Rodriguez, hep-ph/0103307; T. Han and J. Hewett, Phys. Rev. D 60, 074015 (1999); F. del Aguila, J. A. Aguilar-Saavedra, R. Miquel, Phys. Rev. Lett. 82, 1628 (1999); J. A. Aguilar-Saavedra, B. M. Nobre, hep-ph/0210360.

[9] J. A. Aguilar-Saavedra, G. C. Branco, Phys. Lett. B 495, 347 (2000); J. A. Aguilar-Saavedra, Phys. Lett. B 502, 115 (2001); J. A. Aguilar-Saavedra, T. Riemann, hep-ph/0102197.

[10] Koh Abe et al., ACFA Linear Collider Working Group, hep-ph/0109166.

[11] S. Bar-Sharlom and J. Wudka, Phys. Rev. D 86, 3722(2001); Phys. Rev. D 60, 094016 (1999).

[12] See, e.g, Z. Xiong and J. M. Yang, Nucl. Phys. B 628, 193 (2002); C. Bobeth, T. Ewerth, F. Kruger, J. Urban, hepph/0204225; S.Rai Choudhury, Naveen Gaur, hep-ph/0207353; hep-ph/0206128; hep-ph/0205076; hep-ph/0203041;

[13] See, e.g., F. Gabbiani, E. Gabrielli, A. Masiero, L. Silverstrini, Nucl. Phys. B 477, 321 (1996); M. Misiak, S. Pokorski, J. Rosiek, Adv. Ser. Direct. High Energy Phys. 15, 795 (1998); F. Borzumati, C. Greub and T. Hurth, Phys. Rev. D 62, 075005 (2000); T. Besmer, C. Greub and T. Hurth, Nucl. Phys. B 609, 359 (2001).

[14] M. J. Duncan, Nucl. Phys. B 221, 285 (1983); Phys. Rev. D 31, 1139 (1985).

[15] K. Hikasa and M. Kobayashi, Phys. Rev. D 36, 724 (1987).

[16] S. J. Brodsky, T. Kinoshita and H. Terazawa, Phys. Rev. D 4, 1532 (1971); H. Terazawa, Rev. Mod. Phys.45, 615(1973); B. A. Kniehl, Phys. Lett. B 254, 267(1991).

[17] R. Cahn and S. Dawson, Phys. Lett. B 136, 196 (1984); M. Chanowitz and M. K. Gaillard, Phys. Lett. B 142, 85 (1984); G. L. Kane, et. al., Phys. Lett. B 148, 367(1984).

[18] I. F. Ginzburg, et al., Nucl. Instrum219, 5 (1984); V. I. Telnov, Nucl. Instrum. Meth.294, 72 (1990).

[19] Particle Physics Group. Euro. Phys. J. C 15, 274 (2000).

[20] Y. Zeng-Hui, et. al., Euro. Phys. J. C 16, 541 (2000).

[21] J. Yi, et. al., Phys. Rev. D 57, 4343 (1998).

[22] T. Hahn, Nucl. Phys. Proc. Suppl.89, 231 (2000); Acta Phys. Plon. B30, 3469 (1999).

[23] K. J. Abraham, K. Whisnant and B.-L. Young, Phys. Lett. B 419, 381 (1998).

[24] R. Frey et al., FERMILAB-CONF-97-085 (1997); hep-ph/9704243.

[25] J. L. Diaz-Cruz, H. J. He and C. P. Yuan, Phys. Lett. B 530, 179 (2002); H.-J. He, S. Kanemura, C.-P. Yuan, hep$\mathrm{ph} / 0203090$.

[26] D. Atwood, S. Bar-Shalom, G. Eilam and A. Soni, hep-ph/0203200. 
[27] G 't Hooft and M. Veltman, Nucl. Phys. B 44, 189 (1972); A. Denner, Fortschr. Phys. 41, 307 (1993). 